\title{
Key Parameter identification of quad-rotor aircraft system based on CIFER Algorithm
}

\author{
Hao-ran $\mathrm{Qi}^{\mathrm{a}}{ }^{\text {) }}$ Xiao-hui Qi ${ }^{\mathrm{b}}$ \\ Department of Unmanned Aerial Vehicle, Ordnance Engineering College, Shijiazhuang 050003, China \\ a)holidayqihaoran@163.com \\ b)qi-xh@163.com
}

\begin{abstract}
Currently,there are some limitations of twxisting ways of obtaining the key parameter of quad-rotor aircrafts—-mechanism analysis method and experimental method,that the first one is not adapt to varied environment and need a large amount of computation,the second one especially refers to wind tunnel test requires high cost and long operating time.This paper presented a method of combining mechanism analysis method and parameter identification to estimate the key parameters of the quad-rotor,which was easy to realize and has good performance.Firstly,the system model of the quad-rotor under the hover condition was build.Then the method based on CIFER software was used to identificate the uncertainly parameters of quad-rotor on the basis of the measured data of the 3-DOF hover simulation platform. The result showed at low frequency this method is effective and accurate in system identification of quad-rotor.
\end{abstract}

Keywords: Parameter, quad-rotor, CIFER

\section{INTRODUCTION}

With the rapid development of new materials, micro-electro-mechanical system and micro rotorcraft flight control technology in recent years, micro rotorcraft unmanned aerial vehicle (UAV) such as quad-rotor is widely applied in all kinds of military and civilian fields ${ }^{[1]}$. In order to make quad rotor complete specific missions, people need to design its control system, and establish accurate system model ${ }^{[2]}$. To obtain various major parameters of quad-rotor, such as rotor lift coefficient and rotary inertia is the key to achieve successful modeling.

At present, there are mainly two ways to obtain the key parameters of quad-rotor ${ }^{[3]}$. The first one is mechanism analysis. The calculation is conducted through the traditional engineering experience and knowledge of modern aerodynamics. However, it's difficult to balance the accuracy, real-time performance and computation complexity. The second one is the test method, including wind tunnel test method and parameter identification method. To obtain the key parameters of quad-rotor through wind tunnel test method has disadvantage of long test cycle and high cost ${ }^{[4]}$. Therefore, to estimate system parameter value through operation processing by adopting the method of combining mechanism 
analysis and parameter identification test, is an the effective means to obtain the key parameters of quad-rotor, and build accurate model .

Many scholars have studied quad-rotor parameter identification methods, and achieved some results. Literature [5]estimates values through constrained linear least squares method, and obtains the aerodynamic parameters of UAV through iterative operation according to a certain constraint conditions. Literature[6] identifies the parameters of spacecraft control function with maximum likelihood method, and obtains good effect. However, the identification results based on the least squares are vulnerable to the measurement noise; the maximum likelihood method involves quite complex computation; artificial neural network tends to fall into local optimal solution. Compared to the above methods, the key parameters in the frequency domain identification is more suitable for quad-rotor system with high order dynamic characteristics, and low signal-to-noise ratio of flight data.

Based on the complex features of quad-rotor, the paper first carried on theoretical analysis and established the dynamics model of experimental platform, and then identified the key parameters through CIFER frequency domain algorithm.

\section{ESTABLISHMENT OF MODEL}

In the paper, system model was established based on the existing quad-rotor laboratory experiment platform, and the unknown key parameters were extracted. Besides, the flight data were collected with the platform, and the parameter identification test was carried out.

\section{Introduction to Experiment Platform}

The semi-physical simulation environment of quad-rotor was based on quad-rotor platform of Canadian Quanser., as shown in Figure 1.

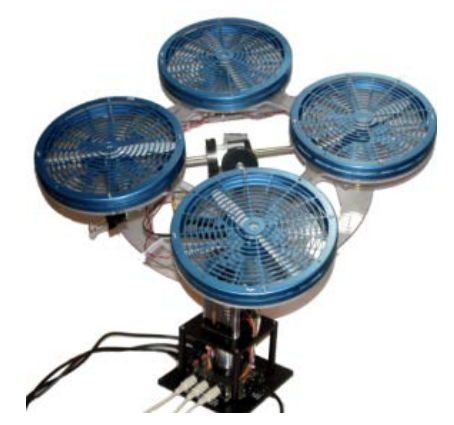

FIGURE 13 -DOF hover simulation platform of quad-rotor

3 DOF quad-rotor platform consists of quad-rotor body and base. Its four rotor wings are distributed in the shape of "cross". After determining the direction of the nose, it can be labeled with four parts, namely, "front”, "rear”, "left” and "right”; “front” and "rear” rotate counterclockwise, while "left" and "right rotor clockwise.

\section{Experiment Platform Modeling}

In order to establish dynamics model of quad-rotor platform, ground coordinate system $O_{I} X_{I} Y_{I} Z_{I}$ and body coordinate system $O X Y Z$ are first established, as shown in Figure 2. 


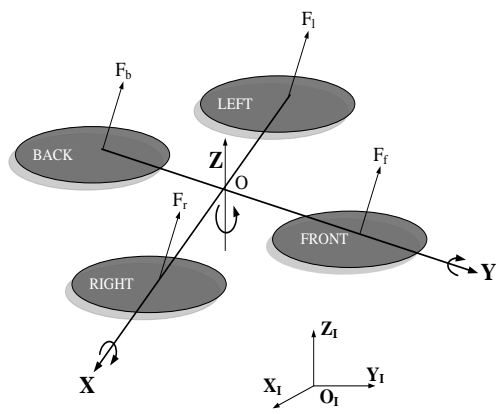

FIGURE 2 Establish of coordinate system

The key of setting up accurate mathematical model of quad-rotor lies in the analysis of rotor aerodynamic model. According to blade element theory, $T, D$ and $L$ respectively are the lift force, resistance and side lurch force produced by a single rotor; $\Omega$ denotes rotor wing speed. The overall coefficient of rotorlift force, resistance and side lurch force are as follows:

$$
\left.\begin{array}{l}
\Gamma_{T}=\frac{1}{2} \rho \pi r_{m}^{2} C_{T} r_{m}^{2} \\
\Gamma_{D}=\frac{1}{2} \rho \pi r_{m}{ }^{2} C_{D} r_{m}^{2} \\
\Gamma_{L}=\frac{1}{2} \rho \pi r_{m}{ }^{2} C_{L} r_{m}^{2}
\end{array}\right\}
$$

In above equations, $C_{T}, C_{D}$ and $C_{L}$ are lift force coefficient, resistance coefficient and side lurch force coefficient of rotor wing respectively; $\rho$ is air density; $r_{m}$ is propeller radius.

The total torque of the rotor wing on the platform is as follows:

$$
\boldsymbol{M}=\left[\begin{array}{l}
M_{x} \\
M_{y} \\
M_{z}
\end{array}\right]=\left[\begin{array}{l}
d \Gamma_{T}\left(\Omega_{B}{ }^{2}-\Omega_{F}{ }^{2}\right) \\
d \Gamma_{T}\left(\Omega_{R}{ }^{2}-\Omega_{L}{ }^{2}\right) \\
\Gamma_{L}\left(\Omega_{F}{ }^{2}+\Omega_{R}{ }^{2}-\Omega_{L}{ }^{2}-\Omega_{B}{ }^{2}\right)
\end{array}\right]
$$

In above equations, $\Omega_{F}, \Omega_{R}, \Omega_{L}$ and $\Omega_{B}$ are respectively the revolving speed of front, right, left and rear motor; $d$ is the rotor wing arm length. In the experimental platform, the square of the rotor wing speed is positively correlated with the voltage of the corresponding motor; and the amount of input control for quad-rotor is the voltage of four motors. Therefore, equation (2) in the platform can be expressed in the following form:

$$
\boldsymbol{M}=\left[\begin{array}{l}
M_{x} \\
M_{y} \\
M_{z}
\end{array}\right]=\left[\begin{array}{l}
d K_{T}\left(V_{B}-V_{F}\right) \\
d K_{T}\left(V_{R}-V_{L}\right) \\
K_{L}\left(V_{F}+V_{R}-V_{L}-V_{B}\right)
\end{array}\right]
$$

In above equations, $V_{R}, V_{L}, V_{F}$ and $V_{B}$ are the voltage of four motors; $K_{T}$ denotes coefficient between the motor voltage and lift force; $K_{L}$ is coefficient between the motor voltage and axial torque.

$\boldsymbol{\omega}=\left[\begin{array}{lll}p & q & r\end{array}\right]^{T}$ is used to express the three attitude angular velocity of quad-rotor platform rotating round rotation axis under the body coordinate system. I denotes the rotational inertia the motor produced on the direction of rotation. According to the Newton-Euler equation, the dynamics equations of quad-rotor platform can be obtained as follows :

$$
I \dot{\omega}+\omega \times I \omega=M+M_{e}+M_{l}
$$

In above equation, $\omega \times I \omega$ is the rolling effect produced by body rotation; $M$ is the torque of 
four motors, and used as the input of the control system; $M_{e}$ is the friction torque turn on rotation bearing; $M_{l}$ is the rotor wing of lurch force torque.

Through the above analysis, we still needed to know the parameter $d, K_{T}, K_{L}$ and before establishing system model.

\section{IDENTIFICATION EXPERIMENT}

Due to the high symmetry of quad-rotor structure, the aerodynamic parameter and other performance of four motors are merely different, and can be thought to be approximately equal. Thus, after the reasonable analysis of one of the motor, the performance parameters of the other three motors can be deduced.

Frequency domain identification is generally suitable for linear time invariant system, so linearization was needed on the established nonlinear model near horizontal balance point. When quad-rotor platform is at hover state $L \approx 0$ and the value of the friction torque $M_{e}$ could be neglected,and $\omega \times I \omega=0$. Therefore, the dynamics equation of quad-rotor will be:

$$
\text { I } \dot{r}=K_{T} L\left(V_{R}-V_{L}\right)
$$

It was expressed in the form of state space. By selecting quantity of state $x=\left[\begin{array}{ll}\theta & \dot{\theta}\end{array}\right]^{T}$ output quantity $y=\theta$, where $\theta$ is quad-rotor roll angle. Then, the following equations could be obtained:

$$
\left\{\begin{array}{l}
\dot{x}=\left[\begin{array}{ll}
0 & 1 \\
0 & 0
\end{array}\right] x+\left[\begin{array}{c}
0 \\
I^{-1} L K_{T}
\end{array}\right]\left(V_{R}-V_{L}\right) \\
y=\left[\begin{array}{ll}
1 & 0
\end{array}\right] x
\end{array}\right.
$$

The Laplace transform form of system frequency response matrix $T(s)$ is as follows:

$$
Y(s)=T(s) U(s)
$$

In Equation 9, $U(s)$ and $Y(s)$ are the Laplace transform of input and output signals. The estimation matrix of frequency response matrix can be expressed with the following form:

$$
\hat{T}_{c}=\frac{G_{x y}(f)}{G_{x x}(f)}
$$

In above equation, $G_{x y}(f)$ and $G_{x x}(f)$ are cross-power spectrum function and auto-power spectrum function respectively. System identification aimed to make the estimate matrix of frequency response matrix conform to the actual data matrix $\hat{T}_{c}$ to the largest degree. Design function was $W_{r}$; the model used for identification was output error model. The identification criteria is as follows:

$$
J=\sum_{i=1}^{n_{\rho}} J_{1}=\sum_{i=1}^{n_{s}}\left\{\frac{20}{n_{\omega}} \sum_{i=\omega_{1}}^{\omega_{n}} W_{\gamma}\left[W_{g}\left(\left|\hat{T}_{c}\right|-|T|\right)^{2}-W_{p}\left(\angle \hat{T}_{c}-\angle T\right)^{2}\right]\right\}
$$

Where $W_{\gamma}=1.58\left(1-\mathrm{e}^{-\gamma^{2}}\right)$.

In Equation 9, $n_{\omega}$ denotes the number of frequency point; $\omega_{1}$ and $\omega_{n}$ are the appropriate initial and cut-off frequency; $W_{\gamma}$ is weight function related to coherence function, in which $\gamma^{2}$ represents the coherent value of each frequency point. $W_{g}$ and $W_{p}$ are the weight functions associated with the variance of amplitude and phase; $\hat{T}_{c}$ is the estimation matrix of frequency response matrix.

Frequency domain identification was carried out through identification software CIFER, with its 
basic flow shown in Figure 3.

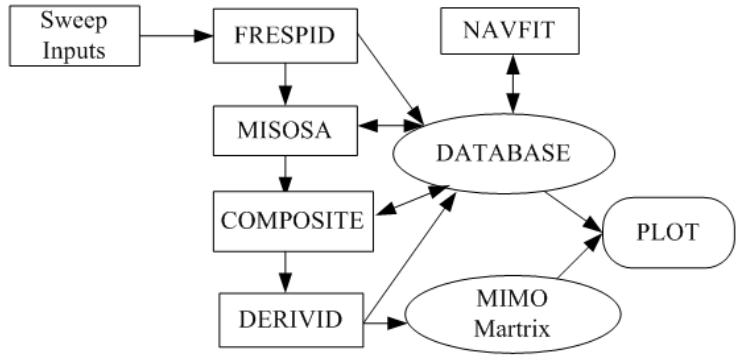

FIGURE 3 Operation flow of CIFER

The steps on the frequency domain identification were as follows:

1. Frequency sweep signals were input to quad-rotor platform, sweep frequency data were collected and the data were processed to make it conform to the actual test requirements. The frequency response of each input and output pair was calculated mainly through Chip-Z transformation.

2. Frequency response and partial coherence needed regularizing through multiple input identification technique.

3.To obtain the most accurate identification estimates, the above spectrum calculation needed processing based on optimized composite window technology. Finally, an independent MIMO complex frequency response estimation matrix $\hat{T}_{c}$ with excellent quality and dynamic range was obtained.

4. Single channel transfer function identification was carried out on the processed data, and response transfer function was obtained.

5.First of all, the stability of the estimation model was analyzed. Then the minimum error of frequency response was reached according to the change of estimate of unknown parameters. Finally, the system state space equation and the values of the unknown parameters that needed identifying were obtained. 6.The frequency domain and time domain of the identification results were verified.

Sweep frequency signal was input to quad-rotor experiment platform, and the design of frequency scanningwas $0.3 \sim 12 \mathrm{rad} / \mathrm{s}$. The system state space equations were established based on system input quantity $u$, quantity of state $X$ and output quantity $y$ :

$$
\left\{\begin{array}{l}
M \dot{x}=F x+G u(t-\tau) \\
y=H x+T \bar{x}
\end{array}\right.
$$

In above equations, $M, F, G, H, T$ and $\tau$ are coefficient matrix and the coefficient that needed solving in CIFER. In the experiment, the rotor wing arm length $l=0.197 \mathrm{~m}$. The key parameters of rotational inertia $I$ and rotor wing lift force coefficient needed identifying. Unknown parameter $I$ and $K_{T}$ were included in the model matrix: $\theta=\left[I, K_{T}\right]$. Based on the initial value of parameters in prior estimate, recognition vector value is constantly changed through iterative process until the $J$ of the cost function reached the minimum.

On the basis of the frequency domain identification process through CIFER package the identification results of the key parameters were extracted, namely, $\hat{I}=0.0492$ and $\hat{K}_{T}=0.1170$. After comparing them with the values ( $I=0.0552$ and $K_{T}=0.1188$ ) given by user manual, it was found that the identification results had small range of error, namely, about $\pm 10 \%$ of error of rotational inertia $\hat{I}$ and about $\pm 1.5 \%$ of error of rotor wing lift force coefficient $\hat{K}_{T}$. Because there was certain error in the parameters given by the user manual of semi-physical quad-rotor simulation platform, the parameters for each independent equipment were not necessarily consistent with them completely.The analysis results showed that the parameters obtained by identification met accuracy 
requirement.

The experimental results are shown in Figure 4. The solid line and dotted line in the graph respectively describe the amplitude and phase frequency response of the model obtained by actual data frequency response and identification of frequency sweep experiment.
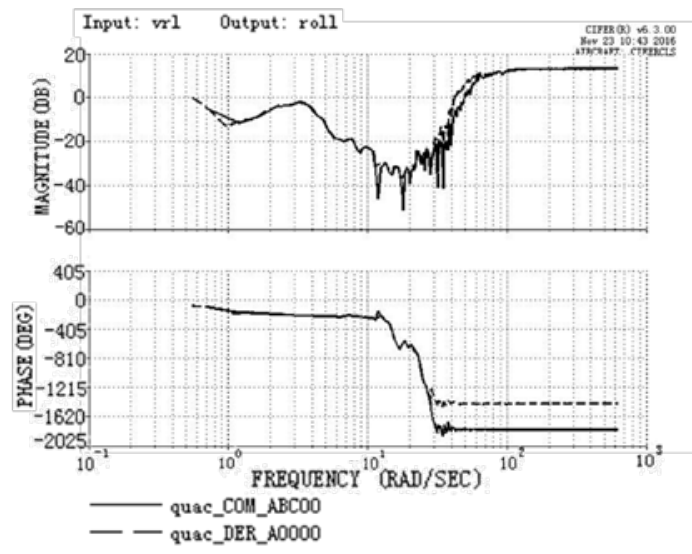

FIGURE 4 The comparison of recognition result and experimental data frequency response

As can be seen from the analysis of above results, the experiment data acquisition frequency was $50 \mathrm{~Hz}$; frequency response amplitude and phase had good effect in low frequency results. There was shacking in the high frequency phase (more than $10 \mathrm{~Hz}$ ), the tracking effect was common possibly because the high frequency phase required additional feedback to maintain the quad-rotor within the scope of the control conditions. the parametric model obtained based on mechanism analysis failed to completely describe the system response. Based on the analysis of identification results, model parameter identification results were more accurate when quad-rotor was closer to hover state.

\section{CONCLUSION}

It is difficult to determine the key parameters of the quad-rotor, therefore the paper designed a kind of frequency domain identification method that combined mechanism analysis and parameter identification, and verified the method through quad-rotor simulation platform. Quad-rotor frequency domain parameter identification through CIFER packages simplifies a lot of formula calculation process, reduce the experiment cost, and is of high maneuverability. Besides, data verification results show that the identification model has high accuracy, and quite precious key parameters.

\section{REFERENCES}

1. Zhao Shulong,An Honglei, Identification of aerodynamic parameter for quadrotor model, J. Journal of Electronic Measurement and Instrument. 08 (2013) 744-749.

2. Zou Yu,Pei Hailong, Study on CIFER Algorithm,a Method for Frequency Identification of Aircraft Model.J.Electronics Optics and Control,05(2010),46-49.

3. Chen Shu,Method analysis of Frequency-domain system identification for a small size unmanned aircraft.,D.Beijing Institute of Technology,2015.

4. Li Hanbign,Wu Dawei,An approach of UAV's aerodynamic parameter identification,J.Flight Dynamics.02(2012)183-188.

5. Liang Xueming,Liang Xiaogeng,A self-organizing fuzzy neural network algorithm for aerodynamic parameter identification,J.Electronics Optics and Control,17(2010),1-4. 
6. Quanser Company. 3 DOF Hover-User Manual[CD]. 2013. 